Pathophysiology Haemostasis and Thrombosis

\section{Risk Assessment and Primary VTE Prevention in the Cancer Patient}

\author{
Mark N. Levine ${ }^{1}$ Agnes Y.Y. Lee ${ }^{2}$ \\ ${ }^{1}$ McMaster University, Faculty of Health Sciences; ${ }^{2}$ Hamilton Civic Hospitals Research Center, Hamilton, Canada
}

Patients with cancer who undergo surgery are at increased risk for venous thromboembolism (VTE). In addition, 'medical' cancer patients who receive chemotherapy, hormonal therapy, and/or radiation are also prone to develop VTE. Finally, cancer patients with central vein catheters (CVC) can develop CVC-associated thrombosis. Prophylaxis with antithrombotic agents can be problematic in cancer patients because they are also at increased risk for anticoagulant-induced bleeding. In this chapter we discuss the evidence and provide recommendations on the prevention of VTE in these three clinical settings.

\section{Surgical Patients}

Among patients with active malignancy, those undergoing surgery have a particularly high risk for thromboembolic disease. In general, prophylaxis with anticoagulant therapy in patients undergoing surgery for malignancy is strongly recommended. However, most of these patients also have a high risk of bleeding. Because malignancy (odds ratio, 1.69) and complex surgery (odds ratio, 1.62) are risk factors for perioperative bleeding in patients receiving heparin prophylaxis [1], it is important that anticoagulant therapy is individualized to balance the competing risks of thrombosis and bleeding.

\begin{tabular}{ll}
\hline KARGER & (c) 2003 S. Karger AG, Basel \\
Fax +41 61 306 1234 & 1424-8832/03/0337-0036\$19.50/0 \\
$\begin{array}{l}\text { E-Mail karger@karger.ch } \\
\text { www.karger.com }\end{array}$ & $\begin{array}{l}\text { Accessible online at: } \\
\text { www.karger.com/journals/pht }\end{array}$
\end{tabular}

\section{General Surgery}

Patients who are undergoing major abdominal or pelvic surgery for malignancy are at high risk for postoperative thrombosis [1]. The reason for the high incidence is multifactorial [2]. In addition to their malignancy, these patients often have concurrent risk factors that increase their risk of thrombosis, including advanced age, debility, long and complicated surgery, and often a prolonged postoperative course.

Kakkar et al. [3] evaluated the risk of thrombosis following major abdominal surgery using ${ }^{125} \mathrm{I}$-fibrinogen leg scanning. This technique is no longer used because of concern over viral transmission and accuracy [4-6]. In addition, many of the thrombi detected with leg scanning were asymptomatic. Kakkar showed that the postoperative rate of DVT was higher in patients with malignancy (41\%) than in patients undergoing surgery for benign disease (26\%).

A significantly higher rate of fatal PE following surgery also has been reported in cancer patients compared to noncancer patients [1]. A subgroup analysis of a large, multicentre study with more than 4,000 patients showed that the rate of fatal $\mathrm{PE}$ was $1.6 \%$ in patients with cancer and only $0.5 \%$ in those without cancer $(\mathrm{P}=0.05)[7,8]$.

Pharmacologic Prophylaxis

The agents used most widely for prophylaxis in surgical patients are unfractionated heparin (UFH) and 
low-molecular-weight heparin (LMWH). Many clinical trials have compared the efficacy and safety of these agents and several large meta-analyses of these trials have not detected any significant differences between these agents in reducing postoperative thrombosis and in anticoagulantrelated bleeding following major abdominal surgery [9-12]. Although many of these trials included cancer patients, they were usually a small subset and the results for the two patient groups were not analyzed or reported separately in many of the studies. In the trials of low-dose UFH versus LMWH that did comment on the differences between cancer and non-cancer patients, a trend toward more thrombotic complications in cancer patients was consistently reported, independent of the prophylaxis agent used [13-17]. In a recent meta-analysis that combined the results for cancer patients from these various trials, Mismetti et al. found no significant difference between LMWH and UFH in cancer patients undergoing surgery for a number of clinically relevant outcomes including symptomatic thromboembolism, major bleeding, transfusions and death [18].

Several trials have evaluated prophylaxis specifically in cancer patients undergoing general surgery. The largest study was performed by the Enoxacan Study Group in Sweden [19]. These investigators compared a LMWH (enoxaparin $40 \mathrm{mg}$ once daily) with UFH (5,000 U 3 times a day) in 1,115 patients having elective curative surgery for cancer of the abdomen or pelvis. According to mandatory bilateral venography used for outcome assessment, the rates of thrombosis were $14.7 \%$ in the enoxaparin group and $18.2 \%$ in the UFH group. These results were not statistically different. There were also no differences in the rates of major bleeding and the 30-day or 3-month mortality.

Taken together, the evidence suggests that once-daily LMWH is as safe and effective as multiple daily injections of UFH for the prevention of postoperative DVT in cancer patients. However, the residual risk of thrombosis with prophylaxis for 5-7 days remains considerable at $10-15 \%$.

To examine whether extended prophylaxis could further reduce the risk of thrombosis, the Enoxacan II Study was conducted. Patients undergoing surgery for abdominal malignancy received 1 week of enoxaparin and then were randomized to enoxaparin or placebo for another 21 days [20]. Bilateral venography was performed at the end of treatment. Extended prophylaxis statistically significantly reduced the rate of DVT from $12 \%$ to $4.8 \%$. However, most of these thrombi were asymptomatic, and the 1-year follow-up showed no difference in overall survival [21]. Therefore, although extended prophylaxis is efficacious in reducing post-operative DVT in cancer patients, additional

Risk Assessment and Primary VTE

Prevention in the Cancer Patient clinical trials are required before extended prophylaxis can be recommended following cancer surgery.

\section{Mechanical Methods}

Devices such as external pneumatic compression and graduated compression stockings are effective in reducing thrombosis after general surgery [22-26]. In a metaanalysis of 11 randomized clinical trials in abdominal, gynecological, and neurosurgery, graduated compression stockings were found to have a risk reduction of $68 \%$ [22]. Although it is effective, the major limitation of external pneumatic compression, is its inconvenience and interference with early mobilization. Therefore, clinicians usually reserve it for patients with active hemorrhage or a high risk of bleeding following surgery. Mechanical devices can also be used in combination with UFH or LMWH in patients with a particularly high risk of thrombosis, e.g., those with a history of VTE, but evidence showing additive or synergistic effects are lacking.

In summary, pharmacologic and mechanical methods of prophylaxis are effective and are recommended in cancer patients who are undergoing general surgery. Thromboprophylaxis should be initiated before surgery and continued throughout the acute, and possibly the extended, postoperative period. Unfractionated heparin and LMWHs appear to be equally safe and effective, but LMWHs have the major advantage of once-daily administration. Graduated compression stockings are effective and may improve the efficacy of pharmacologic prophylaxis alone. External pneumatic compression is an acceptable alternative in patients in whom anticoagulant use is contraindicated. In the cancer patient undergoing general surgery, we recommend once-daily LMWH plus graduated compression stockings.

\section{Neurosurgery}

There is a general reluctance in neurosurgery to use anticoagulant prophylaxis because of the concern for intracranial or intraspinal bleeding. As a result, mechanical methods - such as external pneumatic compression, with or without graduated compression stockings - have typically been the preferred prophylactic measures in these patients $[27,28]$. However, the rate of postoperative thrombosis with compression stockings alone is approximately $30 \%$ based on mandatory bilateral venography $[29,30]$.

Recently, two randomized, placebo-controlled trials compared the combined use of LMWH and graduated compression stockings with stockings alone in patients undergoing elective neurosurgery $[29,30]$. More than $80 \%$ of the patients in each study were having surgery for a brain or spinal cord tumor. In both studies, the added administration 
of a LMWH, starting within 24 hours after surgery, significantly improved on the efficacy of stockings alone without a concomitant increase in the rate of major bleeding. However, despite a relative risk reduction of $30 \%$ to $40 \%$ with LMWH, about $18 \%$ of the patients who received LMWH and stockings still had evidence of thrombosis on mandatory bilateral venography approximately 1 week after surgery. This high residual risk may be due to the potent thrombogenic stimulus generated from large amounts of tissue factor released during neurosurgery, the delay in starting anticoagulant prophylaxis until after surgery, and to the protracted immobilization after surgery.

In contrast, preoperative administration of a LMWH may be associated with an increased risk of intracranial hemorrhage [31]. In an open-label study, patients with an intracranial neoplasm undergoing a neurosurgical procedure were randomized to receive enoxaparin, a sequential compression device, or both. After 66 patients were enrolled, the study was terminated prematurely when three patients in the combined therapy group and one patient in the enoxaparin group experienced clinically significant intracerebral bleeding, and one patient in the enoxaparin group suffered an epidural hematoma. There were no bleeding episodes in the mechanical prophylaxis group.

Based on the results of the two randomized trials, the most recent American College of Chest Physician (ACCP) consensus guidelines, published in 2001, concluded that the combination of a LMWH and a mechanical method may be more effective than either a pharmacological or mechanical method alone [32]. Nonetheless, there is a widespread reluctance to use LMWH within the perioperative period in these patients because of the concern for bleeding. It remains paramount that all cases should be assessed individually and those patients with an increased risk of bleeding should be treated with mechanical prophylaxis alone. Thus, to summarize, for cancer patients undergoing neurosurgery we recommend LMWH commencing no sooner than $24 \mathrm{~h}$ postoperatively, plus mechanical prophylaxis (graduated compression stockings or intermittent pneumatic compression) or mechanical prophylaxis alone.

\section{Gynecological Surgery}

The early studies in this patient group were a series of small, open-label, controlled trials in which women with gynecological cancers were randomized to receive prophylaxis versus no treatment [33-36]. Using ${ }^{125}$ I-fibrinogen leg scanning or impedance plethysmography for screening, these studies found that the incidence of postoperative venous thrombosis following surgery for gynecological malignancy without prophylaxis ranged from $12 \%$ to $35 \%$. Various prophylactic regimens, including low dose unfractionated heparin (UFH) commencing $2 \mathrm{~h}$ preoperatively, UFH commencing $16 \mathrm{~h}$ preoperatively and external pneumatic compression, were found to be effective in reducing this risk by $20-60 \%$.

Other investigators have compared LMWH with UFH in patients undergoing surgery for gynecological malignancy $[37,38]$. In these trials, no difference was detected between LMWH and UFH. The major shortcomings of most of these studies are the insufficient statistical power as a result of small sample sizes and the use of noninvasive leg testing for screening DVT. Nonetheless, the limited evidence demonstrates that the risk of thrombosis following gynecological surgery for cancer is substantial and that prophylaxis with anticoagulant therapy is indicated. The available data are also consistent with the observations in general surgery and suggest that once-daily LMWH administration is likely to be as efficacious and safe as UFH given in multiple injections. For patients undergoing extensive surgery for malignancy, the 2001 ACCP guidelines recommended either low-dose heparin three times a day, with or without compression stockings or intermittent pneumatic compression, or LMWH [32]. For cancer patients undergoing gynecological surgery, we recommend LMWH plus compression stockings in most patients and reserve LMWH plus pneumatic compression for patients at especially high risk for thrombosis (e.g. with a past history of VTE).

\section{Medical Patients}

When considering the prevention of VTE in the medical cancer patient, the physician should be prepared to deal with two main clinical situations: the ambulatory patient who is receiving chemotherapy or radiation and the patient who is bedridden for prolonged periods of time.

Much less data are available on the primary prevention of thrombosis in ambulatory cancer patients compared to prevention after cancer surgery. Levine et al. [39] showed that low-dose warfarin is effective in reducing the rate of thrombosis during chemotherapy. In a double blind, randomized trial, 311 patients with Stage IV breast cancer were given either very low-dose warfarin or placebo while they were receiving chemotherapy. The warfarin dose was $1 \mathrm{mg}$ daily for 6 weeks and was then adjusted to have the INR maintained at 1.3 to 1.9 . The average duration of therapy was 6 months. Seven patients in the placebo group and one in the warfarin group experienced a thrombotic event during follow-up. This relative risk reduction of $85 \%$ was 
statistically significant $(P=0.031)$ and was not associated with an increase in bleeding.

Despite this study, oncologists do not commonly practice prophylaxis with oral anticoagulants during chemotherapy. The most likely reasons are the concern for bleeding and the logistics of laboratory monitoring and dose adjustments, which are frequently required to compensate for changes in the platelet count and interaction with concomitant medications. An alternative is to reserve prophylaxis for high-risk situations (e.g. previous history of VTE or a pelvic mass causing poor venous drainage from the lower limbs). Although there are a number of trials that have evaluated long-term LMWH as secondary prophylaxis in patients with established venous thrombosis, there have been no trials reported evaluating it for long-term primary prevention of VTE.

Similarly, no studies have evaluated long-term prophylaxis in bedridden cancer patients. Low-dose UFH and LMWH have been found to be effective in patients hospitalized with acute medical illnesses (e.g. myocardial infarction and ischemic stroke) [32]. Samama et al. [40] demonstrated that short-term prophylaxis with LMWH in hospitalized medical patients significantly reduced the rate of DVT detected by screening venography. There was a trend, however, for an increase in overall bleeding. Thus, based on these considerations, it would seem reasonable that patients with advanced malignancy who are bedridden would benefit from prophylaxis with low-dose UFH or LMWH. The feasibility and cost-effectiveness of long-term subcutaneous administration, however, remain uncertain.

\section{Catheter-Related Thrombosis}

Long-term indwelling central venous catheters (CVCs) are commonly used in cancer patients for the administration of chemotherapy, parenteral nutrition, and blood products and for facilitating the drawing of blood. One of the complications of CVCs is venous thrombosis related to the catheter. The extent of thrombosis associated with a CVC can involve the catheter tip (ball-valve clot), the length of the catheter (fibrin sheath), the catheterized vessel in the upper limb, the central vasculature of the neck or mediastinum, or a combination of these sites.

Although a number of studies have reported on the incidence of CVC-related thrombosis, the rates have varied between studies and hence the true incidence in cancer patients is uncertain [41]. The discrepancy is due to a number of factors, including the retrospective nature and small size of most of the studies, differences in the populations studied, and variation in the diagnostic tests used to detect CVC-related thrombosis.

Four randomized studies have been performed to evaluate the safety and efficacy of prophylactic anticoagulation in patients with CVCs; two investigated low-dose warfarin and two examined LMWH. The first open-label study using low-dose warfarin was reported in 1990 by Bern et al. [42] who randomized 84 cancer patients to receive $1 \mathrm{mg}$ warfarin daily or no treatment. Mandatory contrast venography was performed at 90 days or sooner if patients had symptoms suggestive of thrombosis. The rate of thrombosis was $37 \%$ in the control group compared to $10 \%$ in the warfarin group $(\mathrm{P}<0.001)$. The majority of the patients with thrombosis were symptomatic by 30 days. There was no difference between the bleeding rates between the two groups, but four patients $(10 \%)$ in the warfarin group required vita$\min \mathrm{K}$ to reverse a prolonged prothrombin time. In contrast, Couban et al. demonstrated no difference between $1 \mathrm{mg}$ of warfarin and placebo in a more recent and larger study with 255 patients [43]. The risk of symptomatic CVC-related thrombosis was approximately $4 \%$ in both groups.

Conflicting evidence has also been reported for LMWH. Monreal et al. [44] performed an open-label randomized study that evaluated low-dose LMWH in patients with CVCs. Patients requiring a port device for chemotherapy infusion were treated with dalteparin 2,500 IU subcutaneously once daily or no prophylaxis. Based on blinded venographic assessment at day 90, patients who received the LMWH had a much lower incidence of catheter-related thrombosis. The study was terminated early by the safety committee because of a large difference in the thrombotic rates between the groups. Of the 16 patients randomized to the LMWH, one $(6 \%)$ developed thrombosis, compared with eight of 13 patients $(62 \%)$ in the control group $(\mathrm{P}=0.002)$. This is contrary to more recent evidence that suggest low-dose LMWH does not reduce the risk of CVC-related thrombosis. Reichardt et al. studied 425 cancer patients with CVCs who were randomized to 16 weeks of dalteparin or placebo [45]. Venography or ultrasound was performed at 16 weeks. The rate of catheter-associated thrombosis was very low in both groups, $3.7 \%$ and $3.4 \%$ respectively. The reasons for the much lower rates of CVCrelated thrombosis reported in recent trials are uncertain, but improved catheter material and advances in insertion techniques may be partially contributory.

Most clinicians do not routinely prescribe thromboprophylaxis in cancer patients with CVCs. In addition to the conflicting data from the above trials, there is a concern about bleeding related to the anticoagulant. Also, it remains uncertain where asymptomatic catheter-related thrombi may 
be clinically relevant in terms of catheter function and lifespan, pulmonary embolism, and long-term complications such as post-phlebitic syndrome. Finally, there is the inconvenience and cost of long-term antithrombotic prophylaxis. Low-dose warfarin therapy requires INR monitoring, and LMWH requires daily subcutaneous injections. For patients with cancer who may need an indwelling CVC for months, these latter limitations are significant quality-of-life issues. On the other hand, CVC-related thrombosis can complicate the clinical management of the cancer patients because of the need for anticoagulant therapy. It therefore follows that prevention of CVC-associated thrombosis is prudent. The 2001 ACCP guidelines recommend low-dose warfarin or LMWH for preventing CVC-related thrombosis; however, in view of the evidence from the recent randomized trials, this recommendation will likely be challenged. Additional randomized trials are required to clarify the many unanswered questions in this clinical setting.

\section{Summary}

Cancer patients undergoing surgery are at high risk of postoperative VTE. Effective methods of prophylaxis, including low-dose UFH, LMWH and mechanical methods are available. The prophylactic method used should be tailored to both the patient's underlying risk of thrombosis and bleeding. There is much less information available on longterm primary prophylaxis in ambulatory cancer patients. One study demonstrated that low-dose warfarin is effective and safe in women with advanced breast cancer. Further research is required evaluating prolonged antithrombotic prophylaxis in the medical cancer population and in patients with central vein catheters.

\section{References}

1 Kakkar AK, Williamson RC: Prevention of venous thromboembolism in cancer patients. Semin Thromb Haemost 1999;25:239-243.

2 Gallus AS: Prevention of post-operative deep leg vein thrombosis in patients with cancer. Thromb Haemost 1997;78:126-132.

3 Kakkar VV, Howe CT, Nicolaides AN, Renney JT, Clarke MB: Deep vein thrombosis of the leg: Is there a 'high risk' group? Am J Surg 1970;120: 527-530.

4 Agnelli G, Radicchia S, Nenci GG: Diagnosis of deep vein thrombosis in asymptomatic high-risk patients. Haemostasis $1995 ; 25: 40-48$.

5 Cruickshank MK, Levine MN, Hirsh J, et al: An evaluation of impedance plethysmography and ${ }^{125}$ I-fibrinogen leg scanning in patients following hip surgery. Thromb Haemost 1989;62: 830-834.

6 Lensing AW, Hirsh J: ${ }^{125}$ I-fibrinogen leg scanning: Reassessment of its role for the diagnosis of venous thrombosis in post-operative patients. Thromb Haemost 1993;69:2-7.

7 Prevention of fatal postoperative pulmonary embolism by low doses of heparin: An international multicentre trial. Lancet 1975;2:45-51.

8 Rahr HB, Sorensen JV: Venous thromboembolism and cancer. Blood Coagul Fibrinolysis 1992;3: 451-460.

9 Palmer AJ, Schramm W, Kirchhof B, Bergemann R: Low molecular weight heparin and unfractionated heparin for prevention of thromboembolism in general surgery: A meta-analysis of randomised clinical trials. Haemostasis 1997;27:65-74.

10 Koch A, Bouges S, Ziegler S, Dinkel H, Daures JP, Victor N: Low molecular weight heparin and unfractionated heparin in thrombosis prophylaxis after major surgical intervention: Update of previous meta-analyses. Br J Surg 1997;84: 750-759.
11 Nurmohamed MT, Rosendaal FR, Buller HR, et al: Low-molecular-weight heparin versus standard heparin in general and orthopaedic surgery: A meta-analysis. Lancet 1992;340:152-156.

12 Leizorovicz A, Haugh MC, Chapuis FR, Samama MM, Boissel JP: Low molecular weight heparin in prevention of perioperative thrombosis. Br Med J 1992;305:913-920.

13 Bergqvist D, Burmark US, Flordal PA, et al: Low molecular weight heparin started before surgery as prophylaxis against deep vein thrombosis: 2500 versus $5000 \mathrm{XaI}$ units in 2070 patients. Br J Surg 1995;82:496-501.

14 Koppenhagen K, Adolf J, Matthes M, et al: Low molecular weight heparin and prevention of postoperative thrombosis in abdominal surgery. Thromb Haemost 1992;67:627-630.

15 The European Fraxiparin Study (EFS) Group: Comparison of a low molecular weight heparin and unfractionated heparin for the prevention of deep vein thrombosis in patients undergoing abdominal surgery. Br J Surg 1988;75:1058-1063.

16 Kakkar VV, Cohen AT, Edmonson RA, et al: Low molecular weight versus standard heparin for prevention of venous thromboembolism after major abdominal surgery. The Thromboprophylaxis Collaborative Group. Lancet 1993;341:259-265.

17 Nurmohamed MT, Verhaeghe R, Haas S, et al: A comparative trial of a low molecular weight heparin (enoxaparin) versus standard heparin for the prophylaxis of postoperative deep vein thrombosis in general surgery. Am J Surg 1995;169:567-571.

18 Mismetti P, Laporte S, Darmon JY, Buchmuller A, Decousus H: Meta-analysis of low molecular weight heparin in the prevention of venous thromboembolism in general surgery. Br J Sur 2001; 88:913-930.
19 ENOXACAN Study Group: Efficacy and safety of enoxaparin versus unfractionated heparin for prevention of deep vein thrombosis in elective cancer surgery: A double blind randomized multicentre trial with venographic assessment. $\mathrm{Br} \mathrm{J}$ Surg 1997;84:1099-1103.

20 Eldor A, Bergqvist D, Agnelli G, et al: Prolonged thromboprophylaxis in patients undergoing abdominal cancer surgery with enoxaparin: The Enoxacan II Study (abstract). Blood 2000;98:706a.

21 Bergqvist D, Agnelli G, Cohen AT, Eldor A, Nilsson PE, Le Moigne-Amrani A, et al: Duration of prophylaxis against venous thromboembolism with enoxaparin after surgery for cancer. N Engl J Med 2002;346:975-980.

22 Wells PS, Lensing AW, Hirsh J: Graduated compression stockings in the prevention of postoperative venous thromboembolism: A meta-analysis. Arch Intern Med 1994;154:67-72

23 Roberts VC, Cotton LT: Prevention of postoperative deep vein thrombosis in patients with malignant disease. Br Med J 1974;1:358-360.

24 Allan A, Williams JT, Bolton JP, Le Quesne LP: The use of graduated compression stockings in the prevention of postoperative deep vein thrombosis. Br J Surg 1983;70:172-174.

25 Clagett GP, Reisch JS: Prevention of venous thromboembolism in general surgical patients: Results of meta-analysis. Ann Surg 1988;208:227-240.

26 Cisek LJ, Walsh PC: Thromboembolic complications following radical retropubic prostatectomy: Influence of external sequential pneumatic compression devices. Urology 1993;42:406-408.

27 Hamilton MG, Hull RD, Pineo GF: Venous thromboembolism in neurosurgery and neurology patients: A review. Neurosurgery 1994;34: 280-296. 
28 Clagett GP, Anderson FA Jr, Geerts W, et al: Prevention of venous thromboembolism. Chest 1998;114:531S-560S.

29 Nurmohamed MT, van Riel AM, Henkens CM, et al: Low molecular weight heparin and compression stockings in the prevention of venous thromboembolism in neurosurgery. Thromb Haemost 1996;75:233-238.

30 Agnelli G, Piovella F, Buoncristiani P, et al: Enoxaparin plus compression stockings compared with compression stockings alone in the prevention of venous thromboembolism after elective neurosurgery. N Engl J Med 1998;339:80-85.

31 Dickinson LD, Miller LD, Patel CP, Gupta SK: Enoxaparin increases the incidence of postoperative intracranial hemorrhage when initiated preoperatively for deep venous thrombosis prophylaxis in patients with brain tumors. Neurosurgery 1998; 43:1074-1081.

32 Geerts WH, Heit JA, Clagett GP, et al: Prevention of venous thromboembolism. Chest 2001;119: $132 \mathrm{~S}-175 \mathrm{~S}$

33 Clarke-Pearson DL, Creasman WT, Coleman RE, Synan IS, Hinshaw WM: Perioperative external pneumatic calf compression as thromboembolism prophylaxis in gynecologic oncology: Report of a randomized controlled trial. Gynecol Oncol 1984; 18:226-232

34 Clarke-Pearson DL, Synan IS, Hinshaw WM, Coleman RE, Creasman WT: Prevention of postoperative venous thromboembolism by external pneumatic calf compression in patients with gynecologic malignancy. Obstet Gynecol 1984;63:92-98.
35 Clarke-Pearson DL, Synan IS, Creasman WT: Anticoagulation therapy for venous thromboembolism in patients with gynecologic malignancy. Am J Obstet Gynecol 1983;147:369-375.

36 Clarke-Pearson DL, DeLong E, Synan IS, Soper JT, Creasman WT, Coleman RE: A controlled trial of two low-dose heparin regimens for the prevention of postoperative deep vein thrombosis. Obstet Gynecol 1990;75:684-689.

37 Fricker JP, Vergnes Y, Schach R, Heitz A, Eber M, Grunebaum L, et al: Low dose heparin versus low molecular weight heparin (Kabi 2165, Fragmin) in the prophylaxis of thromboembolic complications of abdominal oncological surgery. Eur J Clin Invest 1988;18(6):561-567.

38 Heilmann L, von Tempelhoff GF, Kirkpatrick C, Schneider DM, Hommel G, Pollow K: Comparison of unfractionated versus low molecular weight heparin for deep vein thrombosis prophylaxis during breast and pelvic cancer surgery: Efficacy, safety, and follow-up. Clin Appl Thromb Hemost 1998;4:268-273.

39 Levine M, Hirsh J, Gent M, et al: Double blind randomised trial of a very-low-dose warfarin for prevention of thromboembolism in stage IV breast cancer. Lancet 1994;343:886-889.

40 Samama MM, Cohen AT, Darmon JY, et al: A comparison of enoxaparin with placebo for the prevention of venous thromboembolism in acutely ill medical patients. Prophylaxis in medical patients with Enoxaparin Study Group. N Engl J Med 1999;341:793-800.
41 Bona RD: Thrombotic complications of central venous catheters in cancer patients. Semin Thromb Haemost 1999;25:147-155.

42 Bern MM, Lokich JJ, Wallach SR, et al: Very low doses of warfarin can prevent thrombosis in central venous catheters: A randomized prospective trial. Ann Intern Med 1990;112:423-428.

43 Couban S, Goodyear M, Burnell M, Dolan S: A randomized double-blind placebo-controlled study of low dose warfarin for the prevention of symptomatic central venous catheter-associated thrombosis in patients with cancer (abstract). Blood 2002;100(11):2769.

44 Monreal M, Alastrue A, Rull M, et al: Upper extremity deep venous thrombosis in cancer patients with venous access devices - prophylaxis with a low molecular weight heparin (Fragmin). Thromb Haemost 1996;75:251-253.

45 Reichart P, Kretzschmar A, Biakhov M, Irwin D: A Phase III double blind, placebo-controlled study evaluating the efficacy and safety of daily lowmolecular-weight heparin (dalteparin sodium, fragmin) in preventing catheter-related complications in cancer patients with central venous catheters (abstract). Proc Ann Meet Am Soc Clin Oncol 2002;21:1474.
Risk Assessment and Primary VTE

Prevention in the Cancer Patient
Pathophysiol Haemost Thromb

2003;33(suppl 1):36-41 